\title{
QUADRATIC FORMS, RIGID ELEMENTS AND NONREAL PREORDERS
}

\author{
KAZIMIERZ SZYMICZEK AND JOSEPH YUCAS
}

\begin{abstract}
A nonreal preorder of a quaternionic structure $q: G \times G \rightarrow B$ is a subgroup $T \subseteq G$ such that $-1 \in T$ and $-1 \neq t \in T$ implies $D\langle 1, t\rangle \subseteq T$. The basic part of $q$ is defined to be the set $B=\{ \pm 1\} \cup\{a \in G \mid a$ is not 2-sided rigid $\}$. A. Carson and M. Marshall have shown that if $|G|<\infty$ then every nontrivial nonreal preorder $T$ must contain $B$. The main purpose of this note is to extend this result by replacing $|G|<\infty$ with $[G: T]<\infty$.
\end{abstract}

1. Introduction. Let $q$ : $G \times G \rightarrow Q$ be a quaternionic structure in the terminology of [3]. Recall that this means $G$ is a group of exponent two with a distinguished element $-1, Q$ is a set with distinguished point 0 , and $q$ is a surjective mapping satisfying:

(1) $q(a, b)=q(b, a)$;

(2) $q(a,-a)=0$ (here as always, $-a$ means $(-1) a)$;

(3) $q(a, b)=q(a, c) \Leftrightarrow q(a, b c)=0$; and

(4) $q(a, b)=q(c, d) \Rightarrow \exists x \in G$ with $q(a, b)=q(a, x)$ and $q(c, d)=q(c, x)$.

For $a \in G$, the set $D\langle 1, a\rangle=\{b \in G \mid q(-a, b)=0\}$ is a subgroup of $G$ and 1 , $a \in D\langle 1, a\rangle . a \in G$ is said to be rigid if $D\langle 1, a\rangle=\{1, a\}$ and we say that $a$ is 2-sided rigid if both $a$ and $-a$ are rigid. The basic part of $G$ is the set $B=\{ \pm 1\} \cup$ $\{a \in G \mid a$ is not 2-sided rigid $\}$. In [1], $B$ is shown to be a subgroup of $G$.

Notice that for $-1 \neq b \in B, D\langle 1, b\rangle \subseteq B$ (if $x \in D\langle 1, b\rangle \backslash B$ then $-x$ is rigid and $-b \in D\langle 1,-x\rangle$, but $-b \in\{1,-x\}$ is a contradiction). Consider any subgroup $T \subseteq G$ satisfying

(5) if $-1 \neq t \in T$, then $D\langle 1, t\rangle \subseteq T$.

If $-1 \notin T, T$ is just a preorder. We will call a subgroup $T \subseteq G$ a nonreal preorder if $-1 \in T$ and $T$ satisfies (5).

Examples of nonreal preorders are:

(i) subgroups $T \subseteq G$ containing -1 such that $a$ is rigid $\forall a \in T, a \neq-1$;

(ii) subgroups $T \subseteq G$ containing $B$.

Nonreal preorders of type (i) exist if and only if either $1=-1$ or 1 is rigid (the implication $(\Rightarrow)$ is clear, for $(\Leftarrow)$ take $T=\{ \pm 1\})$. A nonreal preorder of this type will be called trivial.

Conjecture (Marshall). Every nontrivial nonreal preorder is of type (ii).

Received by the editors October 29, 1981 and, in revised form, October 15, 1982.

1980 Mathematics Subject Classification. Primary 10C01, $10 \mathrm{C} 05$. 
It follows from [2, Corollary 2.7] that the conjecture is true if $|G|<\infty$. The main concern of this paper will be to prove the conjecture for nontrivial nonreal preorders of finite index in $G$, but first some elementary remarks on nonreal preorders in general.

Proposition 1.1. Let $T \subseteq G$ be a nonreal preorder. If $\phi=\left\langle t_{1}, \ldots, t_{n}\right\rangle\left(t_{1}, \ldots, t_{n} \in\right.$ $T)$ is anisotropic, then $D(\phi) \subseteq T$.

Proof. By induction on $n$. For $n=2$, suppose $\left\langle t_{1}, t_{2}\right\rangle$ is anisotropic and $x \in D\left\langle t_{1}, t_{2}\right\rangle$. Then $x t_{1} \in D\left\langle 1, t_{2} t_{2}\right\rangle$ and $t_{1} t_{2} \neq-1$, hence $x t_{1} \in T$ forcing $x \in T$. In general, if $x \in D\left\langle t_{1}, \ldots, t_{n}\right\rangle$, then $x \in D\left\langle t_{1}, y\right\rangle$ for some $y \in D\left\langle t_{2}, \ldots, t_{n}\right\rangle$. By induction $y \in T$. Now, $y \neq-t_{1}$, else $\phi$ is isotropic, hence $\left\langle t_{1}, y\right\rangle$ is anisotropic and $x \in T$.

Let $s$ denote the level of $q$, i.e., $s$ is the smallest positive integer such that $-1 \in D(s\langle 1\rangle)$, if no such $s$ exists, we let $s=\infty$.

COROLlaRY 1.2. $D(s\langle 1\rangle) \subseteq \cap T$, where $T$ ranges over all nonreal preorders of $G$.

Of course, if $s=\infty$, by $D(s\langle 1\rangle)$ we mean $\cup_{n=1}^{\infty} D(n\langle 1\rangle)$.

Notice that $\cap T$ is itself a nonreal preorder and, hence, it is the smallest nonreal preorder of $G$. It would be interesting to know necessary and sufficient conditions under which $D(s\langle 1\rangle)=\cap T$.

Let us, in passing, give a constructive description of $\cap T$. For any subset $S \subseteq G$, let $T_{1}(S)=S$ and inductively define

$$
T_{i}(S)= \pm \mathrm{gp}\left[\cup\left\{D\langle 1,-z\rangle \mid 1 \neq z \in T_{i-1}(S)\right\}\right]
$$

where $\operatorname{gp}(A)$ denotes the group generated by $A$.

Notice that $T_{i-1}(S) \subseteq T_{i}(S)$; thus $T(S)=\cup_{i} T_{i}(S)$ is a subgroup of $G$. In fact $T(S)$ is the smallest nonreal preorder of $G$ containing $S$. It follows then that the smallest nonreal preorder of $G$ is $\cap T=T(\{-1\})$.

As in [3, Theorem 5.19] we prove

Proposition 1.3. If $T \subseteq G$ is a nonreal preorder then the restriction $q_{T}$ of $q$ to $T \times T$ is a quaternionic structure. Moreover, $W\left(q_{T}\right)$ is a subring of $W(q)$.

Proof. (1)-(3) are clear. To show that (4) holds suppose $a, b, c, d \in T$ and $q(a, b)=q(c, d)$. Since (4) holds for $q$, there exists $x^{\prime} \in G$ such that $q(a, b)=$ $q\left(a, x^{\prime}\right)$ and $q(c, d)=q\left(c, x^{\prime}\right)$. It follows that $q\left(a, b x^{\prime}\right)=0$, forcing $b x^{\prime} \in D\langle 1,-a\rangle$. If $a \neq 1$, then $b x^{\prime} \in T$, hence $x^{\prime} \in T$. In this case take $x=x^{\prime}$. If $a=1$, take $x=1$. In either case $q(a, b)=q(a, x)$ and $q(c, d)=q(c, x)$. The fact that $W\left(q_{T}\right)$ is a subring of $W(q)$ follows from Proposition 1.1, i.e., by Proposition 1.1 the inclusion map is injective.

2. Rigid elements and binary value sets. As remarked in the introduction the main goal of this section is to show that every nontrivial nonreal preorder of finite index in $G$ is of type (ii). This result will follow after a rather technical discussion of rigid elements and binary value sets. Lemmas 2.1 and 2.3 below are generalized versions of arguments extracted from the proof of [2, Theorem 2.4]. 
For any subgroup $H \subseteq G$ we define sets $X_{i}=X_{i}(H)$ in a manner similar to (6). We let $X_{1}=H$ and inductively define

$$
X_{i}=\bigcup\left\{D\langle 1,-z\rangle \mid 1 \neq z \in X_{i-1}\right\} .
$$

LEMMA 2.1. Let $H$ be a subgroup of $G$ and let $y \in G, y \notin X_{1} X_{3} \cup X_{1} X_{2}^{2}$. Then for $\beta, \gamma \in H, \beta \neq \gamma$, we have $H D\langle 1,-\beta y\rangle \cap H D\langle 1,-\gamma y\rangle=\{1,-y\} H$.

Proof. First let $\alpha \in H$ and suppose $\alpha D\langle 1,-\beta y\rangle \cap D\langle 1,-\gamma y\rangle \neq \varnothing$. Then $\langle 1,-\gamma y\rangle-\alpha\langle 1,-\beta y\rangle \simeq\langle 1,-\alpha\rangle-\gamma y\langle 1,-\alpha \beta \gamma\rangle$ is isotropic so by [3, Corollary 2.12] there exists $w \in D\langle 1,-\alpha \beta \gamma\rangle$ with $\gamma y w \in D\langle 1,-\alpha\rangle$. If $\alpha \neq 1, \alpha \neq \beta \gamma$, then $w$, $\gamma y w \in X_{2}$ so $y=(\gamma)(w)(\gamma y w) \in X_{1} X_{2}^{2}$, contradicting the choice of $y$. Thus $\alpha=1$ or $\alpha=\beta \gamma$. First suppose $\alpha=1$, and $z \in D\langle 1,-\beta y\rangle \cap D\langle 1,-\gamma y\rangle$. Then $z \in$ $D\langle 1,-\beta y\rangle \cap D\langle 1,-\gamma y\rangle \subseteq D\langle 1,-\beta \gamma\rangle \subseteq X_{2}$; thus $z \in X_{2}$ and $\beta y \in D\langle 1,-z\rangle$. If $z \neq$ 1 then $\beta y \in X_{3}$ so $y=\beta(\beta y) \in X_{1} X_{3}$, again contradicting the choice of $y$. Consequently, $z=1$ and $\alpha D\langle 1,-\beta y\rangle \cap D\langle 1,-\gamma y\rangle=\{1\}$. Now suppose $\alpha=\beta \gamma$. Here,

$$
\begin{aligned}
\alpha D\langle 1,-\beta y\rangle \cap D\langle 1,-\gamma y\rangle & =\beta \gamma D\langle 1,-\beta y\rangle \cap D\langle 1,-\gamma y\rangle \\
& =-\gamma y(D\langle 1,-\beta y\rangle \cap D\langle 1,-\gamma y\rangle)=-\gamma y\{1\}
\end{aligned}
$$

by the case $\alpha=1$. It follows that $H D\langle 1,-\beta y\rangle \cap D\langle 1,-\gamma y\rangle=\{1,-\gamma y\}$. Now

$$
\begin{aligned}
H D\langle 1,-\beta y\rangle \cap H D\langle 1,-\gamma y\rangle & =H(H D\langle 1,-\beta y\rangle \cap D\langle 1,-\gamma y\rangle) \\
& =H\{1,-\gamma y\}=H\{1,-y\} .
\end{aligned}
$$

This proves the lemma.

LEMMA 2.2. Let $H$ be a subgroup of $G$ and suppose $y \in G, \beta \in H$.

(i) If $y \notin X_{2}$ then $\langle 1,-y\rangle$ represents at most one element from each coset of $G$ $\bmod H$.

(ii) If $y \notin X_{1} X_{3} \cup X_{1} X_{2}^{2}$ and $z \in D\langle 1,-y\rangle, z \neq 1$, $-y$, then for each $\alpha \neq 1$ in $H$, $D\langle 1,-\alpha y\rangle \cap z H=\varnothing$.

Proof. (i) Suppose $x \in G, 1 \neq \alpha \in H$ and $x, x \alpha \in D\langle 1,-y\rangle$. Then $\alpha=x(x \alpha) \in$ $D\langle 1,-y\rangle$ so $y \in D\langle 1,-\alpha\rangle \subseteq X_{2}$, a contradiction.

(ii) By Lemma 2.1, $H D\langle 1,-y\rangle \cap D\langle 1,-\alpha y\rangle \subseteq\{1,-y\} H$. Suppose there is $\beta \in H$ such that $z \beta \in D\langle 1,-\alpha y\rangle$. Then $z \beta \in\{1,-y\} H$. If $z \beta \in H$ then $z \in H$, hence $y \in D\langle 1,-z\rangle \subseteq X_{2}$, a contradiction. If $z \beta \in-y H$, then $z H=-y H$. But by (i), $z=-y$, again a contradiction.

LEMma 2.3. Let $H$ be a subgroup of $G$ and let $y \in G,-y \notin X_{1} X_{2}$. Further, suppose $y$ is rigid and $-1 \neq \alpha \in H$ is a nonrigid element with $D\langle 1, \alpha\rangle \subseteq H$. If $\alpha^{\prime} \in D\langle 1, \alpha\rangle$ then $\alpha^{\prime} y$ is rigid.

Proof. Let $\rho=\left\langle 1, \alpha, \alpha^{\prime} y, \alpha \alpha^{\prime} y\right\rangle \simeq\langle 1, \alpha, y, \alpha y\rangle$.

$$
\begin{aligned}
D(\rho) & =\bigcup\{D\langle r, \alpha s\rangle \mid r, s \in D\langle 1, y\rangle\} \\
& =D\langle 1, \alpha\rangle \cup D\langle 1, \alpha y\rangle \cup D\langle y, \alpha\rangle \cup D\langle y, \alpha y\rangle \\
& =D\langle 1, \alpha\rangle\{1, y\} \cup D\langle 1, \alpha y\rangle\{1, y\} .
\end{aligned}
$$


Since a group cannot be a union of two proper subgroups we are reduced to considering two cases.

Case 1. $D\langle 1, \alpha\rangle\{1, y\} \subseteq D\langle 1, \alpha y\rangle\{1, y\}=D(\rho)$. Here we show $\alpha$ is rigid, contradicting our hypothesis. Let $\beta \in D\langle 1, \alpha\rangle \subseteq H$. If $\beta \in D\langle 1, \alpha y\rangle$ then $-\alpha y \in$ $D\langle 1,-\beta\rangle$; thus $\beta=1$, else $-\alpha y \in X_{2}$, forcing $-y \in X_{1} X_{2}$. If $\beta \in D\langle y, \alpha\rangle$ then $-y \alpha \in D\langle 1,-\alpha \beta\rangle$. Consequently, $\beta=\alpha$, else $-y \alpha \in X_{2}$ again. This shows $\alpha$ is rigid.

Case 2. $D\langle 1, \alpha y\rangle\{1, y\} \subseteq D\langle 1, \alpha\rangle\{1, y\}=D(\rho)$. Here we show $\alpha^{\prime} y$ is rigid as desired. Let $z \in D\left\langle 1, \alpha^{\prime} y\right\rangle \subseteq D(\rho)=D\langle 1, \alpha\rangle\{1, y\}$. If $z \in D\langle 1, \alpha\rangle$ then $z \in H$. But $-\alpha^{\prime} y \in D\langle 1,-z\rangle$, hence $z=1$, else $-\alpha^{\prime} y \in X_{2}$, forcing $-y \in X_{1} X_{2}$. If $z \in$ $D\langle y, \alpha y\rangle$ then $z y \in D\langle 1, \alpha\rangle \subseteq H$. Consequently, $z \in y H$. Now $z, \alpha^{\prime} y \in D\left\langle 1, \alpha^{\prime} y\right\rangle$ and $-\alpha^{\prime} y \notin X_{2}$ so $z=\alpha^{\prime} y$ by Lemma 2.2(i). Therefore $\alpha^{\prime} y$ is rigid.

Proposition 2.4. Let $T \subseteq G$ be a nontrivial nonreal preorder and suppose $y \in G \backslash T$ is rigid. If $t \in T \cap B$ then ty is rigid.

Proof. Assume first that $t \neq \pm 1$. If $t$ is nonrigid then taking $H=T$ and $\alpha=\alpha^{\prime}=t$ in Lemma 2.3 yields $t y$ is rigid. If $t$ is rigid then $-t$ is nonrigid. Let $t_{1} \in D\langle 1,-t\rangle, t_{1} \neq 1,-t$. Then $t \in D\left\langle 1,-t_{1}\right\rangle$ and by Lemma 2.3 again with $\alpha=-t_{1}$, $\alpha^{\prime}=t$ we get $t y$ is rigid. If $t=1, t y$ is rigid by hypothesis so suppose $t=-1$. If there is a nonrigid element $t^{\prime} \in T \cap B, t^{\prime} \neq \pm 1$, then as above with $-t^{\prime}$ playing the part of $t,-t^{\prime} y$ is rigid. Taking $y=-t^{\prime} y$ and $\alpha=\alpha^{\prime}=t^{\prime}$ in Lemma 2.3 yields $-y=\left(t^{\prime}\right)\left(-t^{\prime} y\right)$ is rigid; thus suppose $T \cap B=\{ \pm 1\}$. Either $1=-1$ or $D\langle 1,1\rangle=\{1,-1\}$. If $1=-1$, it is clear. If $D\langle 1,1\rangle=\{1,-1\}$, then taking $\alpha=1, \alpha^{\prime}=-1$ in Lemma 2.3 again gives us $-y$ is rigid.

COROLlaRY 2.5. Let $T \subseteq G$ be a nontrivial nonreal preorder. Every rigid element in $G \backslash T$ is 2-sided rigid.

THEOREM 2.6. Let $T \subseteq G$ be a nontrivial nonreal preorder. It $[G: T]<\infty$ then $B \subseteq T$.

Proof. Assume there is an element $b \in B \backslash T$. Consider the set $b T$. By Corollary 2.5, no element in $b T \cap B$ is rigid. For each element $z \in b T \cap B$, let $b_{z} \in D\langle 1, z\rangle$, $b_{z} \neq 1, z$. By Lemma 2.2(ii), the mapping $b T \cap B \rightarrow G / T$ via $z \rightarrow \bar{b}_{z}$ is injective, hence $b T \cap B$ is finite. Now $|T \cap B||b(T \cap B)|=|b T \cap B|<\infty$. Hence, $T \cap B$ is a finite nontrivial, nonreal preorder. Consequently, there exists a nonrigid element $-1 \neq t \in T \cap B$ with $|D\langle 1, t\rangle|<\infty$. By [2, Corollary 2.7], $B \subseteq B \cap T \subseteq T$.

\section{REFERENCES}

1. L. Berman, C. Cordes and R. Ware, Quadratic forms, rigid elements, and formal power series fields, J. Algebra 66 (1980), 123-133.

2. A. Carson and M. Marshall, Decomposition of Witt rings, preprint.

3. M. Marshall, Abstract Witt rings, Queen's Papers in Pure and Appl. Math., no. 57, Queen's Univ., Kingston, Ont., 1980.

Department of Mathematics, Silesian University, Katowice, Poland

Department of Mathematics, SOUTHeRn Illinois University, CaRbondale, Illinois 62901 